Open Access

\title{
Quantitative analysis of mRNA expression levels and DNA methylation profiles of three neighboring genes: FUS1, NPRL2/G21 and RASSF1A in non-small cell lung cancer patients
}

Dorota Pastuszak-Lewandoska ${ }^{1 *}$, Jacek Kordiak ${ }^{2 \dagger}$, Monika Migdalska-Sęk , Karolina H. Czarnecka ${ }^{1}$, Adam Antczak ${ }^{3}$, Paweł Górski ${ }^{4}$ Ewa Nawrot ${ }^{1}$, Justyna M. Kiszałkiewicz' ${ }^{1}$ Daria Domańska and Ewa Brzeziańska-Lasota

\begin{abstract}
Background: Tumor suppressor gene (TSG) inactivation plays a crucial role in carcinogenesis. FUS1, NPRL2/G21 and RASSF1A are TSGs from LUCA region at 3p21.3, a critical chromosomal region in lung cancer development. The aim of the study was to analyze and compare the expression levels of these 3 TSGs in NSCLC, as well as in macroscopically unchanged lung tissue surrounding the primary lesion, and to look for the possible epigenetic mechanism of TSG inactivation via gene promoter methylation.

Methods: Expression levels of 3 TSGs and 2 DNA methyltransferases, DNMT1 and DNMT3B, were assessed using real-time PCR method (qPCR) in 59 primary non-small cell lung tumors and the matched macroscopically unchanged lung tissue samples. Promoter methylation status of TSGs was analyzed using methylation-specific PCRs (MSP method) and Methylation Index (MI) value was calculated for each gene.

Results: The expression of all three TSGs were significantly different between NSCLC subtypes: RASSF1A and FUS1 expression levels were significantly lower in squamous cell carcinoma (SCC), and NPRL2/G21 in adenocarcinoma (AC). RASSF1A showed significantly lower expression in tumors vs macroscopically unchanged lung tissues. Methylation frequency was 38-76 \%, depending on the gene. The highest MI value was found for RASSF1A (52 \%) and the lowest for NPRL2/G21 (5\%). The simultaneous decreased expression and methylation of at least one RASSF1A allele was observed in $71 \%$ tumor samples. Inverse correlation between gene expression and promoter methylation was found for FUS1 ( $r s=-0.41$ ) in SCC subtype. Expression levels of DNMTs were significantly increased in 75-92\% NSCLCs and were significantly higher in tumors than in normal lung tissue. However, no correlation between mRNA expression levels of DNMTs and DNA methylation status of the studied TSGs was found.

Conclusions: The results indicate the potential role of the studied TSGs in the differentiation of NSCLC histopathological subtypes. The significant differences in RASSF1A expression levels between NSCLC and macroscopically unchanged lung tissue highlight its possible diagnostic role in lung cancer in situ recognition. High percentage of lung tumor samples with simultaneous RASSF1A decreased expression and gene promoter methylation indicates its epigenetic silencing. However, DNMT overexpression doesn't seem to be a critical determinate of its promoter hypermethylation.
\end{abstract}

Keywords: Non-small cell lung cancer, Gene expression, Promoter methylation, Tumor suppressor gene, DNA methyltransferase

\footnotetext{
* Correspondence: dorota.pastuszak-lewandoska@umed.lodz.pl

${ }^{\dagger}$ Equal contributors

'Department of Molecular Bases of Medicine, Medical University of Lodz, Pomorska 251, C-5, 92-213 Lodz, Poland

Full list of author information is available at the end of the article
} 


\section{Background}

The development of non-small cell lung cancer (NSCLC) is associated with molecular changes in more than 20 genes localized in different chromosomal regions. The most frequent molecular event in lung cancer pathogenesis is allele loss (loss of heterozygosity, LOH) on short arm of chromosome 3 (3p), in multiple, so called critical regions. $\mathrm{LOH}$ analyses in $3 \mathrm{p}$ indicate two frequently affected regions (FARs) within 3p21.3: LUCA (lung cancer region) in the centromeric region (3p21C) and AP20 (Alu-PCR clone 20 region) in the telomeric region $(3 \mathrm{p} 21 \mathrm{~T})$, which contain loci of multiple tumor suppressor genes (TSGs) [1]. Inactivation of TSGs is the key event in carcinogenesis and involves two steps, each of them affecting one allele. Most frequently, one TSG allele is lost due to $\mathrm{LOH}$ and the other - to mutation, or, alternatively, epigenetic inactivation via gene promoter hypermethylation. The studies on $\mathrm{LOH}$ analysis in lung cancer indicated that $3 \mathrm{p}$ allele loss is nearly universal, shows a "discontinuous LOH pattern" involving multiple, discrete sites, and appears to occur firstly in the 600-kb 3p21.3 region [2]. The reported incidence of $\mathrm{LOH}$ in LUCA region varied, ranging from 10 to $74 \%$, however, it was always higher in squamous cell carcinoma (SCC) than in adenocarcinoma (AC), up to $96 \%$ and $50 \%$, respectively [1-3]. Additionally, low mutation rate, below $10 \%$, found in lung cell lines and primary tumors, highlighted the significance of epigenetic modifications of TSGs in lung carcinogenesis $[4,5]$.

Epigenetic mechanisms, which include DNA methylation, post translational modifications to core histones, microRNA (miRNA) and long non-codingRNA (lncRNA) regulation [6], play a crucial role in regulation of gene expression by affecting chromatin accessibility. The best known epigenetic modification in human is DNA methylation. Cancer-related aberrant DNA methylation pattern, e.g., hypermethylation of promoter sequences of TSGs, provide a range of opportunities for risk assessment, early detection, disease progression and prognosis, as well as therapeutic stratification and post-therapeutic monitoring of cancer. In lung carcinogenesis, among the potential DNA methylation biomarkers, the hypermethylation of APC, CDH1, CDH13, DAPK1, FHIT, MGMT, p16INK4a, RAR $\beta$, RASSF1A, RUNX3 and SHOX2 is the most frequently reported $[5,7,8]$. However, among the ever increasing number of new studies there are also those that negate the previous conclusions [9].

DNA methylation is an enzymatic process mediated by DNA methyltransferases (DNMTs). DNMT1, possessing a 7 - to 21- fold preference for hemimethylated DNA than unmethylated DNA, is the primary enzyme responsible for copping methylation patterns, i.e., for its maintenance [10]. DNMT3 family enzymes (-3A and $-3 \mathrm{~B})$ exhibit de novo methylation activity, as they have similar affinities for both unmethylated and hemi-methylated DNA substrates, and affect the methylation status of normally unmethylated CpG sites [10]. Additionally, a cooperation between DNMT3 family and DNMT1 has been shown in cancer [11]. DNMTs not only play a pivotal role in carcinogenesis, being responsible for DNA methylation, but also seem to be promising molecular bio-markers for cancer diagnosis and therapy [12].

The pre-specified hypothesis tested in the study was that the expression levels of 3 selected TSGs from LUCA region (FUS1, NPRL2/G21 and RASSF1A) were decreased in primary non-small cell lung cancer with promoter hypermethylation as the responsible epigenetic mechanism of their silencing. To investigate the factors involved in TSG hypermethylation in NSCLC, we attempted to determine whether DNMT1 and DNMT3B RNA expression levels correlated with the hypermethylation of the promoters of the studied tumor suppressor genes. We tried to elucidate the role of the studied TSGs in early lung carcinogenesis and cancer progression.

\section{Materials and methods}

The study has been approved by the Ethical Committee of the Medical University of Lodz, Poland, no. RNN/ 140/10/KE. Written informed consent was obtained from each patient.

\section{Characterization of the lung tissue samples and patients clinical characteristics}

The study involved the group of 59 patients with diagnosis of NSCLC, treated in the University Clinic of Pneumology and Allergology of $\mathrm{I}^{\text {st }}$ Chair of Internal Diseases of Medical University of Lodz and in the Department of Thoracic Surgery, General and Oncologic Surgery, Medical University of Lodz, Poland, between July 2010 - March 2013. In NSCLC patients, during the planned surgery (lobectomy or pneumonectomy), tissue fragments (100-150 mg) were obtained from the center of primary lesion and the adjacent noncancerous $(10 \mathrm{~cm}$ distant from the primary lesion), macroscopically unchanged tissue (conventional "normal" sample). Immediately after resection, lung tissue samples were placed in a stabilization buffer RNAlater ${ }^{\odot}$. Each tissue sample was divided into smaller parts (30-50 mg) for individual analysis, and frozen at $-80^{\circ} \mathrm{C}$.

The resected tissue samples were post-operatively histhopathologically evaluated and classified according to the AJCC staging as well as TNM classification (pTNM). Based on histopathological assessments, the group of patients was subdivided in relation to NSCLC subtypes, i.e., squamous cell carcinoma (SCC), adenocarcinoma (AC) and large cell carcinoma (LCC). Relevant clinical and pathological characteristics of the patients 
with NSCLC included in this study are summarized in Table 1. All cases were primary tumors without chemoor radiotherapy treatment.

The design of our study assumed the analysis of gene expression in two study groups: the tumor and the surrounding normal lung tissue, to compare the both type of tissue. The more so, because there are reports indicating no significant differences between cancerous and non-cancerous tissue in regard to some TSG expression [13]. On the other hand, accumulating evidence point to genetic/epigenetic changes occurring in normal tissue adjacent to tumor (the so called "field cancerization" process), which are widely described in literature in various cancer types and highlights the importance of early abnormalities in carcinogenesis $[14,15]$.

\section{RNA extraction, real-time PCR (qPCR method)}

Total RNA was extracted from primary lung cancer and macroscopically unchanged lung tissues, using Universal RNA Purification Kit (Eurx, Poland), according to the manufacturer's recommendations. The qualitative and quantitative assessments of RNA samples were determined using RNA 6000 Pico/Nano LabChip kit (Agilent Technologies, USA) in Agilent 2100 Bioanalyzer (Agilent, USA).

Complementary DNA (cDNA) was transcribed from 100 ng of total RNA, using a High-Capacity cDNA
Reverse Transcription Kit (Applied Biosystems, USA). Reverse transcription (RT) master mix contained: 10x RT buffer, 25x dNTP Mix (100 mM), 10x RT Random Primers, MultiScribe ${ }^{\mathrm{Tu}}$ Reverse Transcriptase, RNase Inhibitor and nuclease-free water, in a total volume of $20 \mu \mathrm{l}$. RT reactions were performed in the following conditions: $10 \mathrm{~min}$ at $25^{\circ} \mathrm{C}, 120 \mathrm{~min}$ at $37{ }^{\circ} \mathrm{C}$, then $5 \mathrm{~s}$ at $85{ }^{\circ} \mathrm{C}$ for, and $4{ }^{\circ} \mathrm{C}$ hold.

The relative expression of the studied genes was assessed in qPCRs using Micro Fluidic Cards, the so called TLDA (TaqMan ${ }^{\circ}$ Low Density Custom Arrays) plates, with pre-loaded selected assays (Applied Biosystems, USA): FUS1-Hs00200725_m1, NPRL2/G21$\mathrm{Hs} 00198012 \mathrm{~m} 1, \quad R A S S F 1 A-\mathrm{Hs} 00200394 \mathrm{~m} 1$, DNMT1Hs00945875_m1， DNMT3B-Hs00171876_m1. ESD (Hs00382667_m1) was used as a reference gene and RNA isolated from normal lung tissue (Human Lung Total RNA, Ambion ${ }^{\circ}$, USA) served as calibrator samples.

All qPCRs were performed using 7900HT Fast RealTime PCR System (Applied Biosystems, USA) with RQ software (TaqMan Relative Quantification Assay software). The reaction mixture contained: $50 \mu \mathrm{l}$ cDNA (50 ng) and $50 \mu \mathrm{l} \mathrm{TaqMan}^{\circ}$ Universal Master Mix (Applied Biosystems, USA). The PCR program was as follows: initial incubation $2 \mathrm{~min}$ at $50{ }^{\circ} \mathrm{C}$, AmpliTaq Gold ${ }^{\circ}$ DNA polymerase activation at $94.5^{\circ} \mathrm{C}$ for $10 \mathrm{~min}$, then 40 two-step cycles $30 \mathrm{~s}$ at $97^{\circ} \mathrm{C}$ and $60 \mathrm{~s}$ at $59.7^{\circ} \mathrm{C}$.

Table 1 Clinicopathological features of the studied NSCLC group

\begin{tabular}{|c|c|c|}
\hline Analyzed variables & NSCLC patients & \\
\hline \multirow[t]{2}{*}{ Gender (n, \%) } & Women $(24,41 \%)$ & \\
\hline & Men $(35,59 \%)$ & \\
\hline \multirow[t]{3}{*}{ Age $(n, \%)$} & Mean $61 \pm 7.62$ & $\leq 60$ yrs $(14,24 \%)$ \\
\hline & & $61-70$ yrs $(30,51 \%)$ \\
\hline & & $>70$ yrs $(15,25 \%)$ \\
\hline \multirow[t]{4}{*}{ Smoking status and smoking history ( $\mathrm{n}, \%)$} & Smokers (54, $92 \%)$ & $<40$ Pack Years ${ }^{\mathrm{a}}$ (PYs) $(26,48 \%)$ \\
\hline & current smokers $(31,57 \%)$ & \\
\hline & former smokers $(23,43 \%)$ & $\geq 40$ PYs $(28,52 \%)$ \\
\hline & non-smokers $(5,8 \%)$ & \\
\hline \multirow[t]{3}{*}{ Histopathological type of NSCLC (n, \%) } & $\operatorname{SCC}(34,58 \%)$ & \\
\hline & $\operatorname{NSCC}(25,42 \%)$ & AC $(20,80 \%)$ \\
\hline & & $\operatorname{LCC}(5,20 \%)$ \\
\hline \multirow[t]{3}{*}{ AJCC classification ${ }^{b}(n, \%)$} & I $(11,18 \%)$ & \\
\hline & $\|(21,36 \%)$ & \\
\hline & III $(27,46 \%)$ & \\
\hline \multirow[t]{3}{*}{ Tumor size according to pTNM classification ${ }^{c}(\mathrm{n}, \%)$} & $\mathrm{T} 1(12,20 \%)$ & \\
\hline & T2 (33, $56 \%)$ & \\
\hline & T3/T4 (14, $24 \%)$ & \\
\hline
\end{tabular}

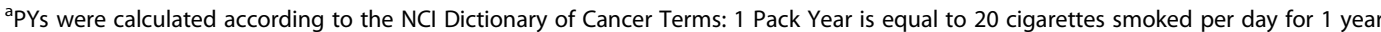
(http://www.cancer.gov/dictionary?CdrID=306)

${ }^{b}$ AJCC - American Joint Committee on Cancer Staging according to the IASCLC Staging Project 7th ed. (2010) Cancer

cpTNM - post-operative Tumor Node Metastasis classification according to the WHO Histological Typing of Lung Tumor 


\section{DNA extraction, bisulfite conversion and} methylation-specific PCRs (MSP method)

The extraction of genomic DNA from NSCLC specimens was performed using a QIAamp DNA Mini Kit (Qiagen, Germany), according to the manufacturer's protocol. To obtain RNA-free genomic DNA, RNase A $(100 \mathrm{mg} / \mathrm{ml})$ was used as optionally indicated in the kit procedure. The quality and quantity of DNA was spectrophotometrically assessed, measuring absorbance at 260/280 nm (Eppendorf BioPhotometer ${ }^{\text {rm }}$ plus, Germany). DNA samples with a $260 / 280 \mathrm{~nm}$ ratio in the range 1.8-2.0 were considered as high quality and selected for further analysis.

Genomic DNA $(1 \mu \mathrm{g})$ was modified with sodium bisulfite, using the CpGenomeTM Turbo Bisulfide Modification Kit (CHEMICON International, Millipore, USA), according to the manufacturer's protocol. Its concentration and purity was spectrophotometrically estimated (Eppendorf BioPhotometer ${ }^{\text {Tix }}$ plus, Germany).

Bisulfite converted DNA was used for methylationspecific polymerase chain reactions (MSPs) to assess methylation status of the studied genes. Primers for MSPs were designed according to the criteria described by Feltus et al. [16], using computer tool (methPrimer v1.1 beta, Li Lab, Department of Urology, USCF) [17]. The set of primers for the studied genes were flanking the $1 \mathrm{~kb} \mathrm{5'}$ region upstream from the translation start point Primer sequences for methylated and unmethylated promoter regions of the studied genes are included in Table 2. MSP master mix contained: $1000 \mathrm{ng}$ DNA, $0.7 \mu \mathrm{M}$ of each primer (Sigma-Aldrich, Germany), $2.5 \mu \mathrm{M}$ dNTPs mix, $2.5 \mu \mathrm{M} \mathrm{MgCl}_{2}$, Hot Start AmpliTaq Gold 360 DNA Polymerase (5U/ $\mu \mathrm{l}), 10 x$ Universal PCR buffer and nuclease-free water, in a total volume of $12.5 \mu \mathrm{l}$. PCR conditions were as follows: initial denaturation at $95{ }^{\circ} \mathrm{C}$ for $5 \mathrm{~min}$, followed by 40 three-step cycles involving denaturation at $95{ }^{\circ} \mathrm{C}$ for $45 \mathrm{~s}$, specific annealing temperature - (see Table 2) - for $45 \mathrm{~s}$ and elongation at $72{ }^{\circ} \mathrm{C}$ for $1 \mathrm{~min}$; the final elongation step was done at $72{ }^{\circ} \mathrm{C}$ for $10 \mathrm{~min}$. The annealing temperatures for each MSP primer were experimentally determined in a set of gradient PCRs. The range of temperatures tested, covering these given in individual primer specifications (Sigma-Aldrich, Germany) were as follows: $50.5-60.5{ }^{\circ} \mathrm{C}$ for FUS1, $52.5-64.2{ }^{\circ} \mathrm{C}$ for NPRL2/G21, and $53-66.5{ }^{\circ} \mathrm{C}$ for RASSF1A.

Positive and negative MSP controls were included in each PCR reaction. CpGenome Universal Methylated DNA (enzymatically methylated human male genomic DNA) served as a positive methylation control and CpGenome Universal Unmethylated DNA (human fetal cell line) was used as a negative control (CHEMICON International, Millipore, USA). As a control for PCR contamination, blank samples with nuclease-free water instead of DNA were used.

The MSP products were electrophoretically separated on $2 \%$ agarose gel and concentrations (ng) of MSP products (U and M DNA alleles) were spectrophotometrically estimated, using DNA1000 LabChip Kit, on Agilent 2100 Bioanalyzer (Agilent Technologies, USA). For each sample, Methylation Index (MI) was assessed using the following formula: peak height of methylated products/(peak height of methylated products + peak height of unmethylated product $), M I=(M) /(M+U)$.

\section{Statistical analysis}

The results of relative expression analysis (RQ values) are presented as medians in each studied group. The comparison of RQ values between cancer and noncancer specimens was performed using Mann-Whitney $U$ test and Kruskal-Wallis test. The same nonparametric tests were applied to compare the differential expressions and methylation status of TSGs between NSCLC subtypes, i.e., SCC, AC and LCC. Spearman's rank correlation coefficient, Mann-Whitney $U$ test and Kruskal-Wallis test were performed to evaluate the relationship between gene expression and methylation levels and clinicopathological parameters (patients' characteristics: age, gender, history of smoking and tumor staging according to pTNM and AJCC classifications). The Newman-Keuls method was used to identify significantly different samples, regarding gene expression and methylation levels. Nonparametric Spearman's criterion was used to calculate the coefficient of correlation between the levels of mRNA expression or

Table 2 Characterization of MSP primers ( $M$ - methylated; $U$ - unmethylated) used in the study. The underlined nucleotides in forward and reverse primers indicate the presence of methylated cytosines in DNA sequence

\begin{tabular}{|c|c|c|c|c|c|c|}
\hline Gene & Forward primer & Primer position & Reverse primer & $\begin{array}{l}\text { Primer } \\
\text { position }\end{array}$ & $\begin{array}{l}\text { Product } \\
\text { length [bp] }\end{array}$ & $\begin{array}{l}\text { Annealing } \\
\text { temp. }\left[\left[^{\circ} \mathrm{C}\right]\right.\end{array}$ \\
\hline \multirow[t]{2}{*}{ FUS1 } & M: TGTTATCGTGGATTAGATATTGTTC & -559 to -482 & M: ACTATATTTTTACGATTACCACGCT & -375 to -349 & 207 & 56.5 \\
\hline & U: TTATI_GTGGATTAGATATTGTIIGT & -557 to -480 & U: ACTATATTITACAATTACCACACCT & -375 to -349 & 205 & 56.5 \\
\hline \multirow[t]{2}{*}{ NPRL2/G21 } & M: GTTCGGTTATTGTTATGGGTAGC & +108 to +131 & M: AACCAATTAAACTCTCGAAAACG & +238 to +261 & 153 & 52.5 \\
\hline & U: GGTITGGTTATTGTTATGGGTAGIG & +107 to +132 & U: ACCAATTAAACTCTCAAAAAACATC & +238 to +261 & 153 & 56.5 \\
\hline \multirow[t]{2}{*}{ RASSF1A } & M: ATATTITTCGATTTGGAGTTITTC & -599 to -573 & M: CTACACTATAACCTACCCATCCTCG & -409 to -384 & 215 & 56.5 \\
\hline & U: TTाTाIGATTGGAGTITITIGT & -599 to -573 & U: TACACTATAACCTACCCATCCTCAC & -410 to -385 & 211 & 57.5 \\
\hline
\end{tabular}


promoter methylation for pairs of studied TSGs and in pairs with methyltransferases. Receiver operating characteristic (ROC) curve was established to discriminate NSCLC and adjacent normal tissue.

$P$-values $<0.05$ were considered statistically significant. All statistical procedures were performed using Statistica for Windows 10.0 software.

\section{Results}

Relative expression levels of the studied TSGs

Relative expression levels of the studied tumor suppressor genes in NSCLC and macroscopically unchanged lung tissue samples were determined using delta-delta $\mathrm{C}_{\mathrm{T}}$ method, and expressed as RQ values adjusted to the expression of ESD (endogenous control) and in relation to the expression level of calibrator (normal lung tissue), for which $R Q=1$.

The obtained RQ values, for the studied TSGs in individual NSCLC samples, are presented in Fig. 1. The simultaneous down regulation of all 3 TSGs was found only in 5.9 \% specimens. Simultaneous decreased expression of RASSF1A and FUS1 was observed in $23.5 \%$ SCC samples, and paired RASSF1A and NPRL2/G21 were simultaneously decreased in $20.9 \%$ SCC and $16 \%$ NSCC samples. Generally, RASSF1A gene showed decreased expression in the majority of lung tumors (especially in SCC subtype), while the expression levels of NPRL2/G21 and FUS1 in most cases were similar to the level of calibrator. Table 3 shows the frequency of NSCLC samples with importantly decreased expression.

The obtained RQ values were correlated with histopathological NSCLC subtypes (SCC, AC, LCC), tumor staging (pTNM, AJCC), patients' age, gender and smoking history, as well as with RQ values of the studied genes in macroscopically unchanged lung tissue samples. Additionally, correlations between all studied genes were analyzed.

Comparing NSCLC histolopathological subtypes, the expression levels of RASSF1A and FUS1 were significantly different, while in case of $N P R L 2 / G 21$ gene, only a tendency was observed. Regarding patients' age, NPRL2/G21 showed significantly lower expression in older patients.

SCC
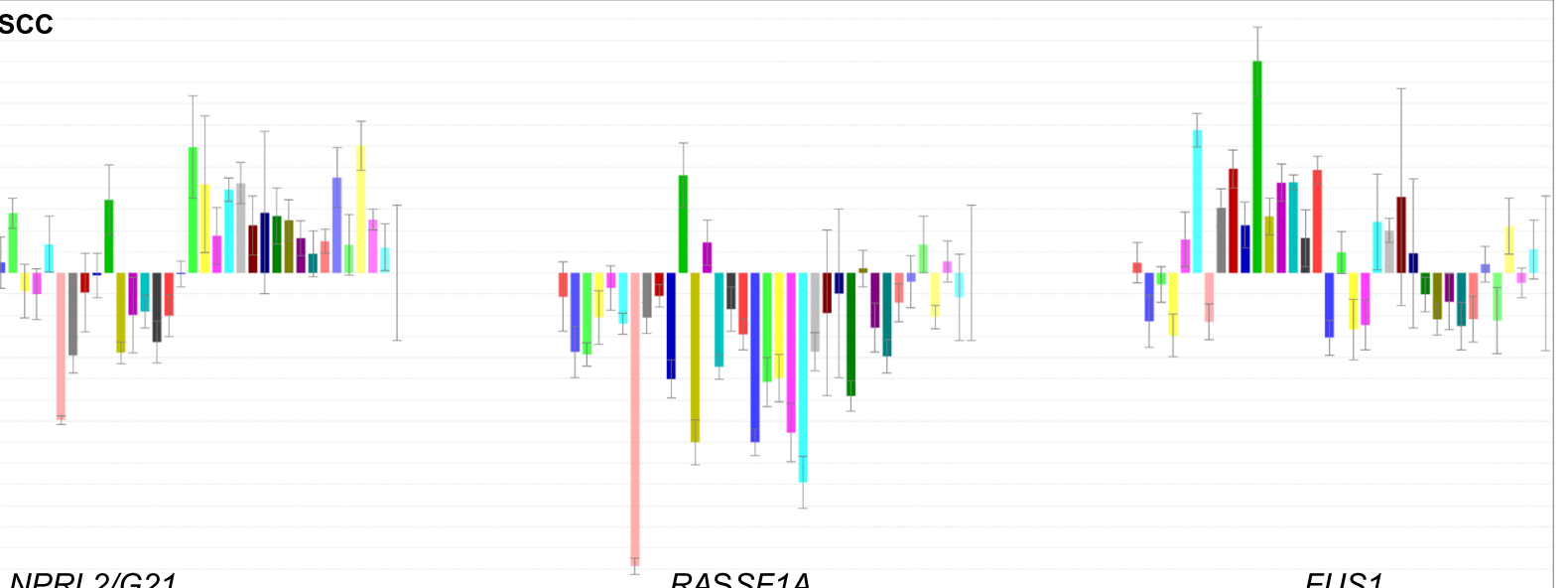

NPRL2/G21

RASSF1A

FUS1

NSCC

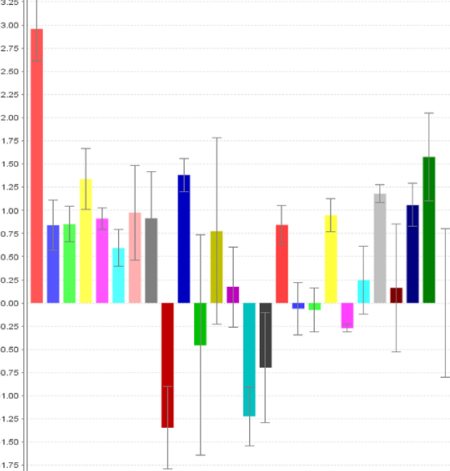

NPRL2/G21
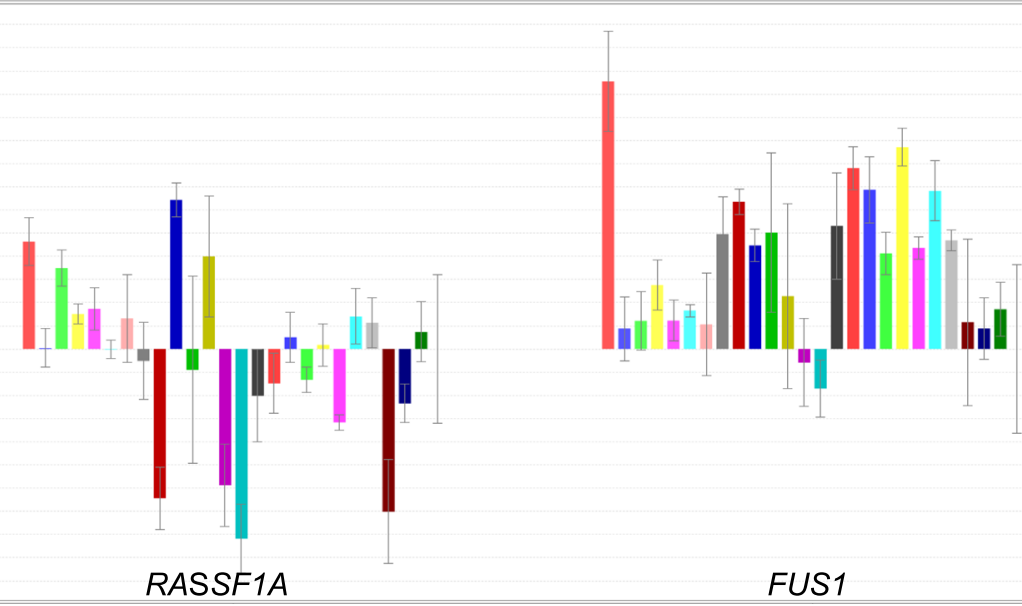

Fig. 1 Relative expression levels of the three TSGs, presented as $\log _{2} R Q$, in the studied NSCLC histopathological subtypes 
Table 3 Frequency of importantly decreased TSG expression $(\mathrm{RQ}<0.5)$

\begin{tabular}{lccccccc}
\hline Gene & \multicolumn{3}{l}{ NSCLC samples $(\mathrm{T})$} & & \multicolumn{3}{c}{ Paired "normal" samples (N) } \\
\cline { 2 - 3 } & Total & SCC & NSCC & & Total & SCC & NSCC \\
& $(n=59)$ & $(n=34)$ & $(n=25)$ & $(n=59)$ & $(n=34)$ & $(n=25)$ \\
\hline RASSF1A & $23.7 \%$ & $29.4 \%$ & $16 \%$ & $5.1 \%$ & $2.9 \%$ & $8 \%$ \\
& $(n=14)$ & $(n=10)$ & $(n=4)$ & $(n=3)$ & $(n=1)$ & $(n=2)$ \\
NPRL2/G21 & $5.1 \%$ & $2.9 \%$ & $8 \%$ & $3.4 \%$ & $2.9 \%$ & $4 \%$ \\
& $(n=3)$ & $(n=1)$ & $(n=2)$ & $(n=2)$ & $(n=1)$ & $(n=1)$ \\
FUS1 & $0 \%$ & $0 \%$ & $0 \%$ & $1.7 \%$ & $2.9 \%$ & $0 \%$ \\
& $(n=0)$ & $(n=0)$ & $(n=0)$ & $(n=1)$ & $(n=1)$ & $(n=0)$ \\
\hline
\end{tabular}

Concerning patients' sex, RASSF1A showed significantly lower expression level in females in NSCC group, and significantly lower expression in males in SCC group. In relation to tumor size (pTNM classification), RASSF1A expression was the lowest in T2 group in total NSCLC group, and similarly in NSCC subgroup. Regarding AJCC classification, statistically significant differences were observed for FUS1 gene in NSCC subtype. Comparing tumor and macroscopically unchanged lung tissue samples, statistically significant differences were found for all genes, however only in case of RASSF1A gene its expression level was significantly lower in tumors. No significant differences were observed in correlation with cigarette smoking. The results are summarized in Table 4.

Newman-Keuls test documented statistical significances between the expression levels of the studied genes in pairs in NSCLC group, as well as in NSCLC histopathological subtypes: statistically significant differences were found between RASSF1A vs NPRL2/G21 $(\mathrm{P}=0.001)$ and RASSF1A vs FUS1 ( $\mathrm{P}=0.00002)$ in total study group, and similarly in SCC subtype, where significant differences were found between RASSF1A vs NPRL2/G21 ( $\mathrm{P}=0.001)$ and RASSF1A vs FUS1 ( $\mathrm{P}=0.0003)$; in AC and NSCC subtypes, the expression levels of RASSF1A and FUS1A were significantly different $(\mathrm{P}=0.03$ and $\mathrm{P}=0.005$, respectively). In all mentioned cases, RASSF1A expression level was significantly lower than those of the other genes. Spearman's rank correlation coefficient revealed positive correlations between genes: RASSF1A and NPRL2/G21 $(\mathrm{P}=0.0001, \mathrm{rs}=0.38)$, RASSF1A and FUS1 $(\mathrm{P}=0.001$, rs $=0.34)$ in NSCLC group; and also between RASSF1A and NPRL2/G21 in AC $(\mathrm{P}=0.02, \mathrm{rs}=0.52)$ and NSCC $(\mathrm{P}=0.002, \mathrm{rs}=0.60)$ group.

ROC curve analysis of RASSF1A expression level as a marker to distinguish NSCLC tissue from macroscopically unchanged lung tissue demonstrated a sensitivity of $61 \%$, specificity of $73 \%$ and accuracy of $67 \%($ AUC $=0.695$ ). Additionally, we analyzed the utility of RASSF1A and FUS1 as potential markers distinguishing NSCLC histopathological subtypes: NSCC and SCC. Analyzed together, the genes yielded a sensitivity of $69 \%$, specificity of $66 \%$ and accuracy of $68 \%$ (AUC =0.714). Separately, ROC curve analysis illustrated for RASSF1A: a sensitivity of $79 \%$, specificity of $64 \%$ and accuracy of $73 \%$ (AUC = 0.729 ); and for FUS1: a sensitivity of $88 \%$, specificity of $56 \%$ and accuracy of $74 \%(\mathrm{AUC}=0.729)$.

\section{Relative expression levels of the studied DNMTs}

Relative expression of two DNA methyltransferases, DNMT1 and DNMT3b, were also analyzed on mRNA level. The expression levels of both DNMTs were increased in tumor samples: median RQ value for DNMT3B was 3.81 and for DNMT1 1.38. Regarding the frequency of samples with increased DNMT expression, DNMT1 was overexpressed in $75 \%$ NSCLCs $(68 \%$ SCC, $76 \%$ NSCC) and DNMT3B in $92 \%$ NSCLCs (91\% SCC, $92 \%$ NSCC).

Correlations were analyzed between the expression levels of the studied DNMTs and histopathological NSCLC subtypes (SCC, AC, LCC), tumor staging (pTNM, AJCC), patients' age, gender and smoking history, as well as in relation to RQ values of the studied DNMTs found in macroscopically unchanged lung tissue samples. DNMT3B expression level was significantly higher in younger patients ( $\leq 60$ vs $61-70$ years old) in AC subtype (7.14 vs 2.43, $\mathrm{P}=0.04$, Mann-Whitney $U$-test) and in NSCC subtype (7.37 vs 2.43, $\mathrm{P}=0.006$, Mann-Whitney $U$-test), where also the negative correlation was observed ( $\mathrm{rs}=-0.50, \mathrm{P}=0.01$, Spearman's rank correlation coefficient). No statistically significant differences were found in relation to patients' gender and smoking, or tumor size (pTNM classification) and grade (AJCC classification). However, in SCC group DNMT1 revealed a trend $(\mathrm{P}=0.05$, Mann-Whitney $U$ test) toward higher expression in grade I/II vs grade III tumors (1.53 vs 1.10).

The expression levels of both DNMTs were significantly higher in tumors when compared with macroscopically unchanged lung tissue samples, in total NSCLC group and in NSCLC histopathological subgroups, as shown in Table 5.

Additionally, the expression levels of the studied DNA methyltransferases were positively correlated (Spearman's rank correlation coefficient) in all tissue groups: in total NSCLC cohort ( $\mathrm{rs}=0.51, \mathrm{P}=0.0001)$, in SCC ( $\mathrm{rs}=0.51$, $\mathrm{P}=0.002)$, in $\mathrm{AC}(\mathrm{rs}=0.49, \mathrm{P}=0.03)$, in NSCC $(\mathrm{rs}=0.55$, $\mathrm{P}=0.004)$.

There weren't any negative correlations between the levels of expression of the methyltransferases and TSGs.

\section{Methylation status of the studied TSG genes: the qualitative and quantitative assessments}

The results on methylation status of the studied genes were obtained for different numbers of patients, due to 
Table 4 Correlations analyzed between the expression levels of the studied genes and tumor clinicopathological parameters and patients' characteristics

\begin{tabular}{lll}
\hline FUS1 $(n=59)$ & NPRL2/G21 $(n=59)$ & RASSF1A $(n=59)$ \\
\hline Median RQ in NSCLC samples & 1.36 & 0.77 \\
1.35 & & \\
SCC vs AC vs LCC & 1.28 vs 1.79 vs $0.83 P=0.05^{\mathrm{a}}$ & 0.68 vs 1.01 vs $0.86 \mathrm{P}=0.01^{\mathrm{a}}$ \\
1.18 vs 1.83 vs $2.39 \mathrm{P}=0.002^{\mathrm{a}}$ & & \\
SCC vs AC & 1.28 vs $1.84 \mathrm{P}=0.03^{\mathrm{b}}$ & 0.68 vs $1.01 \mathrm{P}=0.002^{\mathrm{b}}$ \\
1.18 vs $1.83 \mathrm{P}=0.004^{\mathrm{b}}$ & & \\
SCC vs NSCC & $P>0.05$ & 0.68 vs $1.01 \mathrm{P}=0.002^{\mathrm{b}}$
\end{tabular}

Age ( $\leq 60$ yrs vs $61-70$ yrs vs $>70$ yrs) - in total NSCLC group; in NSCLC histopathological subgroups
$P>0.05$
In SCC: $61-70$ yrs vs $>70$ yrs
$P>0.05$
1.55 vs $0.99 P=0.001^{b}$

Gender (females vs males) - in total NSCLC group; in NSCLC histopathological subgroups
$P>0.05$
$P>0.05$
In SCC: 1.04 vs $0.64 \mathrm{P}=0.02^{\mathrm{b}}$;
in $A C: 0.86$ vs $1.31 \mathrm{P}=0.01^{\mathrm{b}}$;

in NSCC: 0.86 vs $1.31 \mathrm{P}=0.02^{\mathrm{b}}$

pTNM classification (T1 vs T2 vs T3/4) - in total NSCLC group; in NSCLC histopathological subgroups
$P>0.05$
$P>0.05$
0.80 vs 0.67 vs $0.94 \mathrm{P}=0.04^{\mathrm{a}}$;
$\mathrm{T} 2$ vs $\mathrm{T} 3 / 4 \mathrm{P}=0.01^{\mathrm{b}}$

in NSCC: 1.35 vs 0.75 vs $1.27 \mathrm{P}=0.04^{\mathrm{a}} ; \mathrm{T} 2$ vs T3/4 $\mathrm{P}=0.04^{\mathrm{b}}$

AJCC classification (I vs || vs III) - in total NSCLC group; in NSCLC histopathological subgroups

In NSCC: 2.44 vs 1.20 vs $2.25 \mathrm{P}=0.04^{\mathrm{a}}$; I vs $\| \mathrm{P}=0.02^{\mathrm{b}} \quad P>0.05 \quad P>0.05$

Smoking status (current smokers vs former smokers vs never smokers) - in total NSCLC group; in NSCLC histopathological subgroups

$\begin{array}{lll}P>0.05 & P>0.05 & P>0.05\end{array}$

Smoking history (PYs: $<40$ vs $\geq 40$ ) - in total NSCLC group; in NSCLC histopathological subgroups

$\begin{array}{rlrl}P>0.05 & P>0.05 & P>0.05\end{array}$

Tumor (T) tissue vs macroscopically unchanged lung tissue - in total NSCLC group; in NSCLC histopathological subgroups

in $A C: 1.83$ vs $1.18 \mathrm{P}=0.01^{\mathrm{b}}$; $\quad 1.36$ vs $0.84 \mathrm{P}=0.0003^{\mathrm{b}} ; \quad 0.77$ vs $1.07 \mathrm{P}=0.0003^{\mathrm{b}}$;

in NSCC: 2.13 vs $1.20 \mathrm{P}=0.002^{\mathrm{b}}$

in SCC: 1.28 vs $0.84 \mathrm{P}=0.01^{\mathrm{b}}$; $\quad$ in SCC: 0.68 vs $1.09 \mathrm{P}=0.000002^{\mathrm{b}}$

in $A C: 1.84$ vs $0.84 \mathrm{P}=0.002^{\mathrm{b}}$;

in NSCC: 1.79 vs $0.84 \mathrm{P}=0.009^{\mathrm{b}}$

${ }^{\mathrm{a}}$ Kruskal-Wallis test; ${ }^{\mathrm{b}}$ Mann-Whitney U-test

DNA degradation in several samples: 17 in case of RASSF1A, 3 in cases of NPRL2/G21 and FUS1.

Promoter methylation analysis of the studied genes, performed as MSP reactions, distinguished unmethylated (U) from methylated (M) DNA alleles after electrophoretic separation. Both $\mathrm{U}$ and $\mathrm{M}$ alleles were found in all studied tumor tissue groups. Next, based on spectrophotometric estimation (Agilent 2100 Bioanalyzer), fluorescence units (FU) of MSP products were quantified $(\mathrm{ng} / \mu \mathrm{l})$, according to DNA size marker (DNA ladder, Agilent Technologies, USA) and Methylation Index (MI) was calculated for each gene in all tissue samples.

The MSP reactions revealed the presence of $M$ alleles for all genes, in 38-76\% samples, depending on the

Table $5 \mathrm{RQ}$ values (medians) correlated between tumor vs macroscopically unchanged lung tissue samples, with P value (Mann-Whitney U-test)

\begin{tabular}{llll}
\hline Gene & total NSCLC group & SCC subgroup & AC subgroup \\
\hline DNMT1 & 1.38 vs $0.71 P=0.0001$ & 1.36 vs $0.69 P=0.0001$ & 1.57 vs $0.74 P=0.0001$ \\
DNMT3B & 3.81 vs $1.63 P=0.0001$ & 5.24 vs $1.61 P=0.0001$ & 3.63 vs $1.72 P=0.002$ \\
\hline
\end{tabular}


gene. The frequencies of methylated and unmethylated alleles of all studied TSGs, are shown in Fig. 2. The highest frequency of both methylated alleles was observed for RASSF1A gene $(20 \%)$ and the same gene revealed the lowest number of samples with no methylated alleles (4\%). Generally, the most abundant were samples with the presence of both methylated and unmethylated alleles (38-76\%, depending on the gene).

The calculated MIs indicated gene methylation level, which in tumor samples ranged from 3 to $66 \%$, depending on the gene. Among the studied genes, RASSF1A showed the highest methylation level: $52 \%$ in total NSCLC group, $50 \%$ in SCC, and $66 \%$ in AC; NPRL2/ G21 - the lowest: $5 \%$ in total NSCLC group, $3 \%$ in SCC, and $10 \%$ in AC. Methylation level of FUS1 was $17 \%$ in total NSCLC group, also $17 \%$ in SCC and $10 \%$ in $\mathrm{AC}$.

Statistical analysis (Newman-Keuls test) revealed significantly higher methylation status of RASSF1A than those of other studied genes in all tissue groups. In NSCLC: RASSF1A vs FUS1 ( $\mathrm{P}=0.0001)$, RASSF1A vs NPRL2/G21 $(\mathrm{P}=0.00002)$; $\mathrm{MI}$ values of FUS1 were also significantly higher than NPRL2 methylation status $(\mathrm{P}=0.01)$ in this tissue group. The results are shown in Fig. 3. Similarly in SCC group: RASSF1A vs NPRL2/G21 $(\mathrm{P}=0.00002)$, RASSF1A vs FUS1 $(\mathrm{P}=0.0001)$; and also FUS1 vs NPRL2/ G21 $(\mathrm{P}=0.04)$. In AC: RASSF1A vs NPRL2/G21 $(\mathrm{P}=$ 0.0001), RASSF1A vs FUS1 $(\mathrm{P}=0.0001)$.
Concerning tumor histopathological characteristics, in SCC group FUS1 was significantly higher methylated in stage II $v s$ stage III tumors ( $\mathrm{P}=0.03$, Mann-Whitney $U$ test). No other significant correlations were found between gene methylation and clinicopathological parameters.

\section{Correlation between gene expression level and methylation status}

The decreased expression of RASSF1A was accompanied by promoter methylation in $71 \%$ NSCLC samples, in case of FUS1 it was found in $20 \%$ NSCLC specimens, and only in $5 \%$ NSCLC samples for NPRL2/G21. Statistical analysis revealed the negative correlation between the RQ and MI values for only one gene, FUS1 $(\mathrm{P}=0.02$, Spearman's rank correlation coefficient, $\mathrm{rs}=-0.41)$ in SCC subtype.

Spearman's rank correlation coefficient didn't reveal statistically significant correlations between TSG methylation and DNMT expression $(P>0.05)$.

\section{Discussion}

The aim of our study was to analyze by qPCR and compare the expression levels of 3p TSGs in non-small cell lung cancer samples, as well as in macroscopically unchanged tissue surrounding the primary lesion. To look for the possible epigenetic mechanism of TSG inactivation, gene promoter methylation was assessed, as well as the levels of DNMT1 and DNMT3B RNA expression.

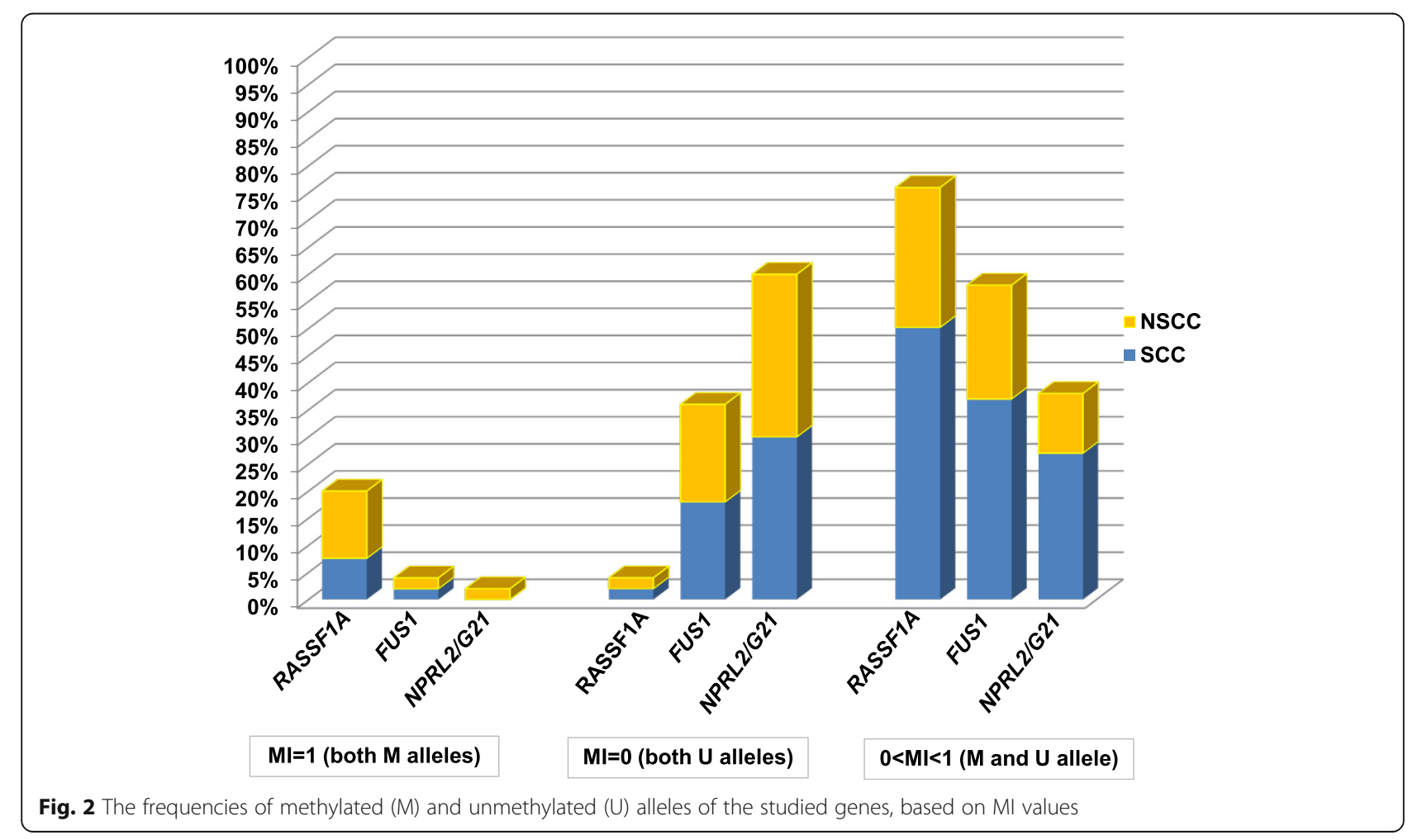




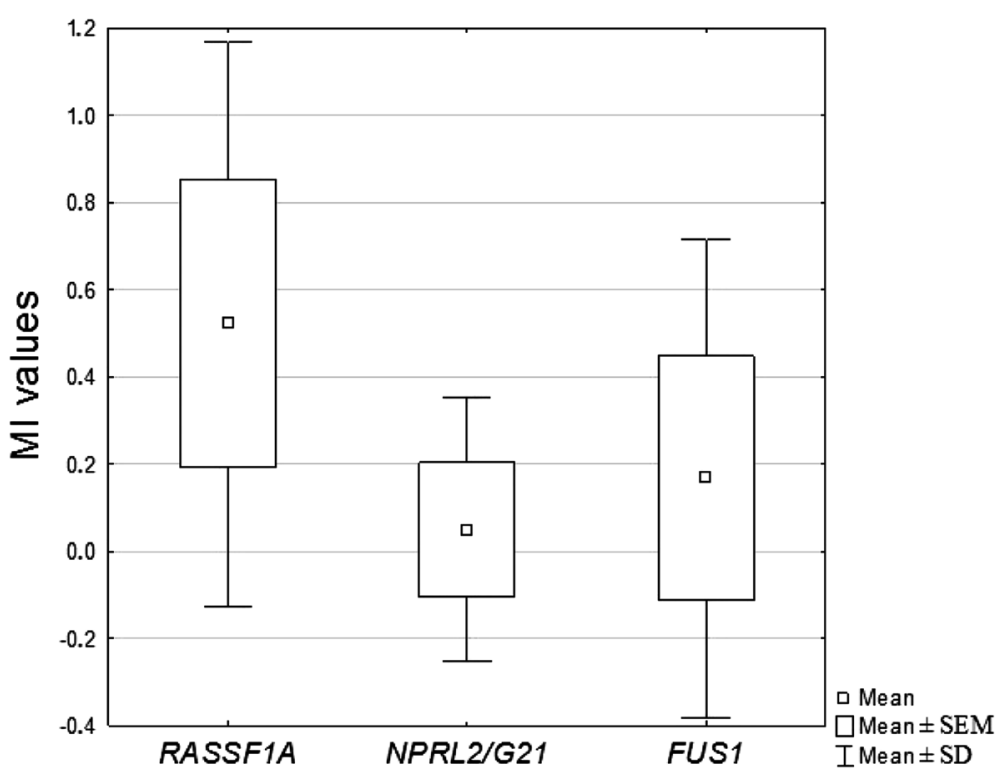

Fig. 3 Box and whisker plot representing mean MI values of the studied genes in NSCLC group

To the best of our knowledge, only few studies were performed to compare expression of several 3p TSGs in the same lung tumor samples [18-22]. In order to investigate it further we chose 3 TSGs and analyzed their expression in lung primary tumors: non-small cell lung cancer (NSCLC), adenocarcinoma (AC), squamous cell lung cancer (SCC) and large cell cancer (LCC). Similarly, few studies have focused so far on simultaneous analysis of methylation status of more than two genes at 3p21.3 locus in primary lung cancer [21, 23, 24].

The chosen TSGs, namely FUS1, NPRL2/G21 and RASSF1A, are located in proximity, in 3p21.3 subregion called LUCA. Such close position could suggest the tendency toward simultaneous decrease of their expression. However, this was not the case in our study. Only few tumor samples showed decreased expression of all three genes. The low levels of promoter methylation of NPRL2/G21 and, to some extent, of FUS1 also didn't designate their epigenetic inactivation in the population studied.

The highest frequency of decreased expression and increased promoter methylation we found for RASSF1A gene. The simultaneous decreased expression and methylation of at least one RASSF1A allele was found in $71 \%$ tumor samples. Tumor suppressor gene, RASSF1A (Ras association domain family member 1), is involved in development or progression in the vast majority of cancers $[4,25,26]$. In NSCLC, RASSF1A decreased expression was found in $67 \%$ samples, as reported by Senchenko et al. [21], although it is usually reported at the level of about $30 \%$ [27]. In our study we confirmed decreased expression of RASSF1A (RQ $<1$ ), and the important gene downregulation $(\mathrm{RQ}<0.5)$ was observed in more than $20 \%$ of NSCLC samples, with higher frequency in SCC group (reaching nearly $30 \%$ ). In paired "normal" lung tissue specimens RASSF1A decreased expression was found only in 3-8 \% samples. The differences between the studied groups, i.e., between NSCLC subtypes and between primary tumor specimens and the surrounding macroscopically unchanged lung tissue samples, were statistically significant (in total study group and in SCC subtype). The highest RASSF1A expression level - similar to that observed in "normal" lung tissue - was found in AC group. Similarly, the increased level of RASSF1A mRNA in lung cancer was also observed by others, who found even 3-to-4 fold increase in AC subtype [21]. RASSF1A gene - as the only one in our analysis - revealed correlations with gender: significantly lower gene expression was found in men in SCC group and in women in NSCC group. Regarding TNM classification, significantly lower RASSF1A expression was observed in T2 tumors as compared with T3/4 NSCLC. It could confirm its role at earlier stages of lung carcinogenesis. However, in other studies, no statistically significant differences were observed with relation to age, smoking history and other cytological and pathological characteristics [21].

Significant differences found between tumor and "normal" lung samples indicate the possible clinical utility of RASSF1A gene expression level as a diagnostic marker. In most studies, the principal cause of RASSF1A loss of expression was tumor-specific RASSF1A promoter hypermethylation, while tumor-associated mutations were rare $[25,26,28]$. In our study, the frequency of RASSF1A 
methylated alleles in total NSCLC group was $76 \%$ and gene methylation level was equal to or exceeded $50 \%$, depending on the histotype. The observed methylation frequency of RASSF1A was in the top of the reported by others $26-87 \%$ range [23, 25, 29-34].

In several reports, RASSF1A methylation was associated with poor prognosis in NSCLC patients [30-32, 35]. We didn't observe any associations with tumor clinicopathological features, such as TNM or AJCC staging, or patients' smoking habits. Similar results were obtained by others [34, 35]. Meta-analysis performed in 2013 confirmed a significant association between RASSF1A methylation and NSCLC, however there were no significant differences in RASSF1A methylation in relation to gender, pathology, TNM stage and smoking behavior among NSCLC cases [36].

It is true that of all the genes in the 3p21.3 critical region RASSF1 has been the most comprehensively studied at the genetic, epigenetic and functional level, and its role as a tumor suppressor gene has been proven. However, the results regarding the utility of RASSF1A epigenetic inactivation as a prognostic marker in NSCLC are still divergent $[9,27,31,33]$. In our study, RASSF1A methylation level was significantly higher than that of other studied genes, however, lack of any other associations does not entitle us to decisive conclusions.

Another gene localized in LUCA region, FUS1 (fused in sarcoma), also known as TUSC2 (tumor suppressor candidate 2) is recently identified TSG, involved in apoptosis. Loss or reduction of FUS1 protein expression, associated with poorer prognosis, was observed in lung cancers [37-39]. We didn't observe importantly decreased expression of FUS1 on mRNA level in lung tumor samples. Statistically significant differences between NSCLC histotypes and the lowest gene expression level in SCC might suggest FUS1 significance predominantly in squamous cell carcinoma. In NSCC subtype, significantly decreased FUS1expression in stage II $v s$ stage III tumors could indicate its role at early stage of carcinogenesis. However, generally, FUS1 expression level was similar to that observed for calibrator, i.e., normal lung tissue. No aberrant FUS1 mRNA level was also found by other researchers [28]. FUS1 promoter methylation analysis in our study revealed the presence of methylated alleles in $58 \%$ NSCLC samples and rather moderate gene methylation status, ranging from 10 to $22 \%$, depending on histopathological subtype. However, as the only gene in our analysis, FUS1 revealed negative correlation between its expression level and methylation status. Additionally, significantly higher gene promoter methylation level in II vs III tumors in SCC subgroup might support FUS1 possible role at early stage of lung cancer development.

So far, there have not been published any reports on FUS1 promoter hypermethylation in lung cancer, although it was found to be partially methylated in head and neck squamous cell carcinoma [40]. Little is known on molecular mechanisms involved in the regulation of FUS1 expression in primary lung cancer cells. FUS1 mRNA transcripts were found in both normal lung and some lung cancer cell lines, but FUS1 protein was absent in a majority of lung cancer cell lines and NSCLC samples [38]. Researchers indicate the role of 3'-untranslated regions (UTRs) of the FUS1 gene transcript [41] or the influence of another epigenetic mechanisms, e.g., the role of several miRNAs in the down-regulation of FUS1 protein expression in lung cancer cells [42] or aberrant histone modifications [43]. Thus, the inactivation of FUS1 may occur through abnormal translational control of FUS1 mRNA or aberrant post-translational protein modification resulting in its inactivation despite the absence of mutations and in the presence of high levels of transcript mRNA [44].

The physiological role of FUS1 is still poorly known and further investigation of this interesting TSG in lung cancer is required.

NPRL2/G21 (nitrogen permease regulator-like 2 gene), also known as TUSC4 (tumour-suppressor candidate 4), is a tumor suppressor gene commonly expressed in normal tissues, including lung tissue. The study results indicate that NPRL2/G21 is involved in DNA repair, cell cycle control and apoptosis [45]. Senchenko et al. [21] showed significantly decreased gene expression level in $73 \%$ NSCLC cases, but the earlier report described normal NPRL2/21 mRNA expression in most lung cancers [25]. Stop mutations, nonsense deletions and missense mutations were found in this gene $[18,45]$. Epigenetic analyses failed to show gene promoter hypermethylation in cancer cell lines $[43,45]$, and no such studies have been performed so far in primary lung tumors.

In our study, we didn't confirm decreased expression of NPRL2/G21 in lung tumor samples. However, the differences between NSCLC histopathological subtypes were significant, with lower expression level in SCC samples. Although, it was still at the level of calibrator (normal lung tissue). In some studies, increased expression of NPRL2/G21 on mRNA or protein level was also observed [21, 46]. Interestingly, NPRL2/G21 gene - as the only TSG in our study group - showed correlation with age: in SCC samples its expression level was significantly lower in elder patients ( $>70$ years old).

The exact mechanism involved in the possible inactivation of NPRL2/G21 in human cancers has not been elucidated. In our study we didn't observe hypermethylation of NPRL2/G21 promoter region. The frequency of gene methylation in NSCLC samples was very low and its methylation status was significantly lower than that of other studied TSGs.

According to the results obtained by Lerman \& Minna [28], NPRL2/G21 may be one of the haploinsufficient 
genes that predispose to cancer in a hemizygous state and do not show a second mutation - or promoter hypermethylation - in the other allele (wild-type allele). Alternatively, as in the case of other $3 \mathrm{p} 21.3$ tumor suppressors, like RASSF1A and FUS1, such mechanisms as chromosome instability, aneuploidy, altered RNA splicing, or defects in transcriptional, translational, and posttranslational modifications that are common in the 3 p region, may play a role in the inactivation of NPRL1/ G21 in lung tumors.

Although the function of NPRL2/G21 is still unknown, it revealed interesting association in lung cancer: gene decreased expression was conversely correlated with cisplatin sensitivity in NSCLC cell lines [46]. This finding, of significant clinical value, could indicate the possible therapeutic role of $N P R L 2 / G 21$ for enhancing and re-sensitizing non-responders to cisplatin. Therefore, NPRL2/G21 deserves attention and further study in lung cancers, regarding its expression and underlying mechanisms of its possible silencing.

None of the genes revealed correlations with smoking history. It is in accordance with the results of Senchenko et al. [21]. Although smoking is a known risk factor in NSCLC, the lack of correlations with expression levels and methylation status of the studied TSGs could indicate that tobacco smoke targets other than studied genes.

We tested whether the methylation status of tumor suppressor genes was associated with the mRNA expression levels of DNA methyltransferases (DNMTs). The role of DNMT mediated epigenetic alterations in lung cancer development has been the focus of increasing interest in recent years. In our study both enzymes were highly expressed in a coordinate manner in lung tumors positive correlation was observed in all NSCLC histopathological types, as well as in total NSCLC group. In case of both studied DNMTs, we found significantly higher expression levels in lung tumor specimens as compared with macroscopically unchanged tissue samples.

Regarding NSCLC histopathological subtypes, DNMT3B expression was higher in SCC than in NSCC, although it didn't reach statistical significance. DNMT1 revealed trend toward higher expression in grade I/II tumors in SCC group, suggesting its role at early stages of lung carcinogenesis. However, in other studies, deregulated expression of DNMT1 was an independent prognostic factor in SCC [47, 48]. Interestingly, in AC and NSCC subtypes, $D N M T 3 B$ expression levels were significantly higher in patients aged 60 and below as compared with older patients. Elevated DNMT expression in younger patients was more frequent also in other study [48].

We didn't observe any negative correlations between the expression levels of the studied TSGs and methyltransferases. The results of others are also conflicting.
Similarly to our findings, the elevated mRNA levels of DNMT1 and DNMT3B were not significantly associated with hypermethylation of the several TSGs, including RASSF1A, in lung cancer cell lines [49] and in lung primary tumors $[48,50]$. Others found correlations between TSG promoter hypermethylation and DNMT overexpression, but on protein level [47]. Overexpression of DNMT was found to occur earlier than the methylation modifications and complex interactions between several factors in hypermethylation process was highlighted $[48,50]$. So, there is still a need to elucidate the clinicopathological significance of DNA methyltransferases in primary non-small cell lung cancer.

\section{Conclusions}

The results of our study indicate the potential role of the studied genes in the differentiation of NSCLC histopathological subtypes: RASSF1A and FUS1 could be regarded as markers differentiating SCC and NSCC, as their expression levels were significantly lower in squamous cell carcinoma subtype; similar role could be assign to NPRL2/G21 in differentiating SCC and AC subtypes. Although, the most prominent role was documented in case of RASSF1A. The significant differences in its expression level between NSCLC and macroscopically unchanged lung tissue surrounding the primary lesions highlight its possible diagnostic role in lung cancer in situ recognition. High percentage of lung tumor samples with simultaneous RASSF1A decreased expression and gene promoter methylation indicates its epigenetic silencing. However, overexpression of methylating enzymes (DNMT1 and DNMT3B) enzymes was not a critical determinate of tumor-specific promoter hypermethylation of RASSF1A and FUS1, which revealed, respectively, high and moderate methylation frequency in our study.

\section{Competing interests}

The authors declare that they have no competing interests.

\section{Authors' contributions}

$A A$ and $E B-L$ conceived the idea of this project. DP-L and JK participated in experimental design, coordination and execution of the experiments and preparation of the manuscript. JK and AA contributed in biological material sampling. DD, EN and JMK participated in sample handling and performed experiments (MSPS, qPCRS). DP-L, JK, and KHC analyzed and interpreted data. MM-S performed the statistical analysis. EB-L revised the manuscript critically for important intellectual content. PG gave final approval for the submission. DP- $L$ and JK have equally contributed to this work. All authors read and approved the final manuscript.

\section{Acknowledgements}

This work was supported by the grant of the National Science Centre, No. 2011/01/B/NZ4/04966.

\section{Author details}

'Department of Molecular Bases of Medicine, Medical University of Lodz, Pomorska 251, C-5, 92-213 Lodz, Poland. Department of Chest Surgery, General and Oncological Surgery, University Hospital No. 2, Medical University of Lodz, Lodz, Poland. ${ }^{3}$ Department of General and Oncological Pulmonology, Medical University of Lodz, Lodz, Poland. ${ }^{4}$ Department of Pneumology and Allergology, Medical University of Lodz, Lodz, Poland. 
Received: 3 March 2015 Accepted: 5 June 2015

\section{Published online: 26 June 2015}

\section{References}

1. Senchenko VN, Liu J, Loginov W, Bazov I, Angeloni D, Seryogin Y, et al. Discovery of frequent homozygous deletions in chromosome 3p21.3 LUCA and AP20 regions in renal, lung and breast carcinomas. Oncogene. 2004;23:5719-28.

2. Wistuba II, Behrens C, Virmani AK, Mele G, Milchgrub S, Girard L, et al. High resolution chromosome $3 p$ allelotyping of human lung cancer and preneoplastic/preinvasive bronchial epithelium reveals multiple, discontinuous sites of $3 p$ allele loss and three regions of frequent breakpoints. Cancer Res. 2000;60(7):1949-60.

3. Antczak A, Migdalska-Sęk M, Pastuszak-Lewandoska D, Czarnecka K, Nawrot E, Domańska D, et al. Significant frequency of allelic imbalance in $3 p$ region covering RARß and MLH1 loci seems to be essential in molecular non-small cell lung cancer diagnosis. Med Oncol. 2013; doi: 10.1007/s12032-013-0532-9.

4. Zabarovsky ER, Lerman MI, Minna JD. Tumor suppressor genes on chromosome $3 \mathrm{p}$ involved in the pathogenesis of lung and other cancers. Oncogene. 2002;21 (45):6915-35.

5. Heller G, Zielinski CC, Zöchbauer-Müller S. Lung cancer: From single-gene methylation to methylome profiling. Cancer Metastasis Rev. 2010;29:95-107.

6. Gibney ER, Nolan CM. Epigenetics and gene expression. Heredity (Edinb). 2010; doi: 10.1038/hdy.2010.54

7. Guo S, Yan F, Xu J, Bao Y, Zhu J, Wang X, et al. Identification and validation of the methylation biomarkers of non-small cell lung cancer (NSCLC). Clin Epigenetics. 2015; doi: 10.1186/s13148-014-0035-3.

8. Guo S, Tan L, Pu W, Wu J, Xu K, Wu J, et al. Quantitative assessment of the diagnostic role of APC promoter methylation in non-small cell lung cancer. Clin Epigenetics. 2014; doi: 10.1186/1868-7083-6-5.

9. Drilon A, Sugita H, Sima CS, Zauderer M, Rudin CM, Kris MG, et al. A prospective study of tumor suppressor gene methylation as a prognostic biomarker in surgically resected stage I to IIIA non-small-cell lung cancers. J Thorac Oncol. 2014; doi: 10.1097/JTO.0000000000000256.

10. Chen T, Li E. Establishment and maintenance of DNA methylation patterns in mammals. Curr Top Microbiol Immunol. 2006:301:179-201.

11. Rhee I, Bachman KE, Park BH, Jair KW, Yen RW, Schuebel KE, et al. DNMT and DNMT3b cooperate to silence genes in human cancer cells. Nature. 2002;416(6880):552-6.

12. Foulks JM, Parnell KM, Nix RN, Chau S, Swierczek K, Saunders M, et al. Epigenetic drug discovery: targeting DNA methyltransferases. J Biomol Screen. 2012; doi: 10.1177/1087057111421212.

13. Malpeli G, Amato E, Dandrea M, Fumagalli C, Debattisti V, Boninsegna L, et al. Methylation-associated down-regulation of RASSF1A and up-regulation of RASSF1C in pancreatic endocrine tumors. BMC Cancer 2011; doi: 10.1186/ 1471-2407-11-351.

14. Tsay JC, Li Z, Yie TA, Wu F, Segal L, Greenberg AK, et al. Molecular characterization of the peripheral airway field of cancerization in lung adenocarcinoma. PLoS One. 2015; doi: 10.1371/journal.pone.0118132.

15. Dakubo GD, Jakupciak JP, Birch-Machin MA, Parr RL. Clinical implications and utility of field cancerization. Cancer Cell Int. 2007;7:2.

16. Feltus FA, Lee EK, Costello JF, Plass C, Vertino PM. Predicting aberrant CpG island methylation. Proc Natl Acad Sci U S A. 2003;100(21):12253-8.

17. Li LC, Dahiya R. MethPrimer: designing primers for methylation PCRs. Bioinformatics. 2002;18(11):1427-31.

18. Calvo R, West J, Franklin W, Erickson P, Bemis L, Li E, et al. Altered HOX and WNT7A expression in human lung cancer. Proc Natl Acad Sci U S A. 2000:97(23):12776-81.

19. Hesson LB, Cooper WN, Latif F. Evaluation of the 3p21.3 tumour-suppressor gene cluster Oncogene 2007; doi:10.1038/sj.onc.1210547

20. Wang F, Grigorieva EV, Li J, Senchenko VN, Pavlova TV, Anedchenko EA, et al. HYAL1 and HYAL2 inhibit tumour growth in vivo but not in vitro. PLoS One. 2008; doi: 10.1371/journal.pone.0003031.

21. Senchenko VN, Anedchenko EA, Kondratieva TT, Krasnov GS, Dmitriev AA, Zabarovska VI, et al. Simultaneous down-regulation of tumor suppressor genes RBSP3/CTDSPL, NPRL2/G21 and RASSF1A in primary non-small cell lung cancer. BMC Cancer. 2010; doi: 10.1186/1471-2407-10-75.

22. Vageli DP, Zaravinos A, Daniil Z, Dahabreh J, Doukas SG, Spandidos DA, et al. hMSH2 and hMLH1 gene expression patterns differ between lung adenocarcinoma and squamous cell carcinoma: correlation with patient survival and response to adjuvant chemotherapy treatment. Int J Biol Markers. 2013; doi: 10.5301/JBM.2012.9420.
23. Seng TJ, Currey N, Cooper WA, Lee CS, Chan C, Horvath L, et al. DLEC1 and MLH1 promoter methylation are associated with poor prognosis in non-small cell lung carcinoma. Br J Cancer. 2008;99(2):375-82.

24. Ito $\mathrm{M}$, Ito $\mathrm{G}$, Kondo $\mathrm{M}$, Uchiyama M, Fukui T, Mori S, et al. Frequent inactivation of RASSF1A, BLU, and SEMA3B on 3p21.3 by promoter hypermethylation and allele loss in non-small cell lung cancer. Cancer Lett. 2005;225(1):131-9.

25. Dammann R, Li C, Yoon JH, Chin PL, Bates S, Pfeifer GP. Epigenetic inactivation of a RAS association domain family protein from the lung tumour suppressor locus 3p21.3. Nat Genet. 2000;25(3):315-9.

26. Agathanggelou A, Cooper WN, Latif F. Role of the Ras-association domain family 1 tumor suppressor gene in human cancers. Cancer Res. 2005; doi: 10.1158/0008-5472.CAN-04-4088.

27. Hesson LB, Cooper WN, Latif F. The role of RASSF1A methylation in cancer. Dis Markers. 2007;23(1-2):73-87.

28. Lerman MI, Minna JD. The 630-kb lung cancer homozygous deletion region on human chromosome 3p21.3: identification and evaluation of the resident candidate tumor suppressor genes. The International Lung Cancer Chromosome 3p21.3 Tumor Suppressor Gene Consortium. Cancer Res. 2000;60:6116-33.

29. Burbee DG, Forgacs E, Zöchbauer-Müller S, Shivakumar L, Fong K, Gao B, et al. Epigenetic inactivation of RASSF1A in lung and breast cancers and malignant phenotype suppression. J Natl Cancer Inst. 2001;93(9):691-9.

30. Wang J, Lee JJ, Wang L, Liu DD, Lu C, Fan YH, et al. Value of p16INK4a and RASSF1A promoter hypermethylation in prognosis of patients with resectable non-small cell lung cancer. Clin Cancer Res. 2004:10(18 Pt 1):6119-25.

31. Choi N, Son DS, Song I, Lee HS, Lim YS, Song MS, et al. RASSF1A is not appropriate as an early detection marker or a prognostic marker for non-small cell lung cancer. Int J Cancer. 2005;115(4):575-81.

32. Yanagawa N, Tamura G, Oizumi H, Kanauchi N, Endoh M, Sadahiro M, et al. Promoter hypermethylation of RASSF1A and RUNX3 genes as an independent prognostic prediction marker in surgically resected non-small cell lung cancers. Lung Cancer. 2007;58(1):131-8.

33. Niklinska W, Naumnik W, Sulewska A, Kozłowski M, Pankiewicz W, Milewski R. Prognostic significance of DAPK and RASSF1A promoter hypermethylation in non-small cell lung cancer (NSCLC). Folia Histochem Cytobiol. 2009:47(2):275-80.

34. Li W, Deng J, Tang JX. Combined effects methylation of FHIT, RASSF1A and RAR $\beta$ genes on non-small cell lung cancer in the Chinese population. Asian Pac J Cancer Prev. 2014;15(13):5233-7.

35. Kim DH, Kim JS, Ji Yl, Shim YM, Kim H, Han J, et al. Hypermethylation of RASSF1A promoter is associated with the age at starting smoking and a poor prognosis in primary non-small cell lung cancer. Cancer Res. 2003:63(13):3743-6.

36. Liu WJ, Tan XH, Guo BP, Ke Q, Sun J, Cen H. Associations between RASSF1A promoter methylation and NSCLC: a meta-analysis of published data. Asian Pac J Cancer Prev. 2013;14(6):3719-24.

37. Ji L, Roth JA. Tumor suppressor FUS1 signaling pathway. J Thorac Oncol. 2008; doi: 10.1097/JTO.0b013e31816bce65.

38. Prudkin L, Behrens C, Liu DD, Zhou X, Ozburn NC, Bekele BN, et al. Loss and reduction of FUS1 protein expression is a frequent phenomenon in the pathogenesis of lung cancer. Clin Cancer Res. 2008;14(1):41-7.

39. Meng J, Majidi M, Fang B, Ji L, Bekele BN, Minna JD, et al. The tumor suppressor gene TUSC2 (FUS1) sensitizes NSCLC to the AKT inhibitor MK2206 in LKB1-dependent manner. PLoS One. 2013; doi: 10.1371/ journal.pone.0077067.

40. Demokan S, Chuang AY, Chang X, Khan T, Smith IM, Pattani KM, et al. Identification of guanine nucleotide-binding protein $\mathrm{Y}^{-7}$ as an epigenetically silenced gene in head and neck cancer by gene expression profiling. Int J Oncol. 2013; doi: 10.3892/ijo.2013.1808.

41. Lin J, Xu K, Gitanjali J, Roth JA, Ji L. Regulation of tumor suppressor gene FUS1 expression by the untranslated regions of mRNA in human lung cancer cells. Biochem Biophys Res Commun. 2011; doi: 10.1016/ j.bbrc.2011.05.122.

42. Du L, Schageman JJ, Subauste MC, Saber B, Hammond SM, Prudkin L, et al. miR-93, miR-98, and miR-197 regulate expression of tumor suppressor gene FUS1. Mol Cancer Res. 2009; doi: 10.1158/1541-7786.MCR-08-0507

43. da Costa Prando E, Cavalli LR, Rainho CA. Evidence of epigenetic regulation of the tumor suppressor gene cluster flanking RASSF1 in breast cancer cell. lines. Epigenetics. 2011; doi: 10.4161/epi.6.12.18271. 
44. Uno F, Sasaki J, Nishizaki M, Carboni G, Xu K, Atkinson EN, et al. Myristoylation of the fus 1 protein is required for tumor suppression in human lung cancer cells. Cancer Res. 2004;64(9):2969-76.

45. Li J, Wang F, Haraldson K, Protopopov A, Duh FM, Geil L, et al. Functional characterization of the candidate tumor suppressor gene NPRL2/G21 located in 3p21.3C. Cancer Res. 2004;64(18):6438-43.

46. Ueda K, Kawashima H, Ohtani S, Deng WG, Ravoori M, Bankson J, et al. The 3p21.3 tumor suppressor NPRL2 plays an important role in cisplatin-induced resistance in human non-small-cell lung cancer cells. Cancer Res. 2006;66(19):9682-90.

47. Lin RK, Hsu HS, Chang JW, Chen CY, Chen JT, Wang YC. Alteration of DNA methyltransferases contributes to $5^{\prime} \mathrm{CpG}$ methylation and poor prognosis in lung cancer. Lung Cancer. 2007;55(2):205-13.

48. Kim H, Kwon YM, Kim JS, Han J, Shim YM, Park J, et al. Elevated mRNA levels of DNA methyltransferase-1 as an independent prognostic factor in primary nonsmall cell lung cancer. Cancer. 2006;107:1042-9.

49. Sato M, Horio Y, Sekido Y, Minna JD, Shimokata K, Hasegawa Y. The expression of DNA methyltransferases and methyl-CpG-binding proteins is not associated with the methylation status of p14(ARF), p16(INK4a) and RASSF1A in human lung cancer cell lines. Oncogene. 2002;21 (31):4822-9.

50. Vallböhmer D, Brabender J, Yang D, Schneider PM, Metzger R, Danenberg $\mathrm{KD}$, et al. DNA methyltransferases messenger RNA expression and aberrant methylation of $\mathrm{CpG}$ islands in non-small-cell lung cancer: association and prognostic value. Clin Lung Cancer. 2006;8(1):39-44.

\section{Submit your next manuscript to BioMed Central and take full advantage of:}

- Convenient online submission

- Thorough peer review

- No space constraints or color figure charges

- Immediate publication on acceptance

- Inclusion in PubMed, CAS, Scopus and Google Scholar

- Research which is freely available for redistribution 